\title{
Observation of Stabilized Tetragonal Latent Tracks Induced by Single SHI Impacts in Monoclinic Natural Zirconia at Room Temperature
}

\author{
J.H. O'CONnell ${ }^{a}$, M.E. LeE ${ }^{a, *}$ And V.A. SkURATOV ${ }^{b}$ \\ ${ }^{a}$ Centre for HRTEM, Nelson Mandela University, P.O. Box 77000, Port Elizabeth 6031, South Africa \\ ${ }^{b}$ Flerov Laboratory for Nuclear Research, Joint Institute for Nuclear Research, \\ Joliot-Curie 6, 141980 Dubna, Moscow region, Russia
}

\begin{abstract}
In this paper we describe the observed tetragonal nature of individual ion tracks produced in natural monoclinic zirconia as a result of $167 \mathrm{MeV}$ Xe irradiation at room temperature in the non-overlapping track regime. The direct observation by transmission electron microscopy of the tetragonal latent tracks reveals a morphology which were observed to be non-continuous in length and consisting of sections which are approximately $25 \mathrm{~nm}$ in length and an angular separation of $9^{\circ}$ which is consistent with the angular difference for the unit cells of the two phases. The tracks are shown to have rectangular cross-sections which are of the order $2.5 \mathrm{~nm}$. Transmission electron microscopy in situ heating of the tracks reveals that the tetragonal phase transforms back to the monoclinic phase in the range of $350-500^{\circ} \mathrm{C}$ which is well below the bulk transformation temperature of about $1000^{\circ} \mathrm{C}$.
\end{abstract}

DOI: 10.12693/APhysPolA.136.237

PACS/topics: $61.80 .-\mathrm{x}$

\section{Introduction}

Zirconia $\left(\mathrm{ZrO}_{2}\right)$ is a polymorphic oxide that exists in three different crystal structures below its melting point, namely, the high temperature cubic and tetragonal phases, as well as the low temperature monoclinic phase [1]. The tetragonal zirconia lattice has lattice parameters, $a=0.514 \mathrm{~nm}$ and $c=0.526 \mathrm{~nm}$. The crystal structure of the monoclinic phase has cell parameters $a=0.518 \mathrm{~nm}, b=0.521 \mathrm{~nm}$, and $c=0.537 \mathrm{~nm}$ with $\beta=98.8^{\circ}(\beta$ is the angle between the $a$ and $c$ axis) [2].

The tetragonal to monoclinic transformation $(t \rightarrow m)$ is considered to occur via a diffusionless martensitic mechanism. The monoclinic-tetragonal transition spreads over a temperature range of 1100 to $1250^{\circ} \mathrm{C}$ and 1050 to $700^{\circ} \mathrm{C}$ during heating and cooling cycle, respectively. On cooling the phase transformation from tetragonal to monoclinic results in a shear strain of $8-14 \%$ and volume increase of the order of $\approx 5 \%$ [1].

The tetragonal to monoclinic transformation mechanism has received considerable attention and there have been a number of modeling approaches which have been adopted in the interpretation of the phase transformation, namely, the thermodynamic-based models and crystallographic-based models and more recently, the phase field model [2]. The tetragonal phase can not be quenched, however, the tetragonal phase can be stabilized at room temperature in small particles having a dimension less than $30 \mathrm{~nm}$ and is demonstrated by the observation of transformation for crystallites in polycrystalline bulk material, thin layers, or powders [3-5].

*corresponding author; e-mail: michael.lee@mandela.ac.za
The tetragonal phase was shown to be stabilized at room temperature after suitable irradiation of the monoclinic phase, as reported by Sickafus et al. [5], based on TEM observation of polycrystalline monoclinic zirconia irradiated with $340 \mathrm{keV}$ Xe ions to a fluence of $2 \times 10^{17} \mathrm{~cm}^{-2}$ at a temperature of $120 \mathrm{~K}$. The results produced from diffraction studies showed that a bulk transformation took place due to the irradiation, however, they were not able to show unambiguously the difference between tetragonal or cubic transformations. Evidence for the presence of latent tracks was not demonstrated in this work. Recent work by Schuster et al. [7] have demonstrated, using X-ray diffraction (XRD) and the Raman spectroscopy, the quantification of the monoclinic to tetragonal transformation by $\mathrm{U}$ and $\mathrm{Pb}$ ions and found that the transformation occurred at a fluence of $2 \times 10^{12} \mathrm{~cm}^{-2}$ to $5 \times 10^{12} \mathrm{~cm}^{-2}$ which increases to $2.5 \times 10^{13} \mathrm{~cm}^{-2}$ for lighter ions. This "apparent" fluence threshold for the $m \rightarrow t$ transformation is probably due to the detection limit (which is about 1\%) of the applied analytical techniques such as XRD and the Raman spectroscopy. Other techniques used to calculate the ion damage to zirconia were ionoluminescence (IL), cathodoluminescence (CL), and Rutherford backscattering spectrometry/channeling (RBS/C) techniques which were used in combination with molecular dynamics (MD) simulations and phenomenological modelling [8]. However, all the above mentioned techniques were applied to materials irradiated to high fluences. It is therefore apparent that alternative techniques are required to observe the $m \rightarrow t$ transformation at lower fluences and/or with irradiation as a result of lighter ions. The ideal and most reliable analytical technique for this study is clearly the direct observation of latent tracks by transmission electron microscope (TEM) [9]. 


\section{Experimental}

Sections of natural zirconia were irradiated with $167 \mathrm{MeV} \mathrm{Xe}$ ions at the FLNR cyclotron complex in Dubna, Russia to a fluence of $2 \times 10^{10} \mathrm{~cm}^{-2}$ at room temperature. TEM lamellae were prepared in crosssection and plan view geometry using an FEI Helios Nanolab focussed ion beam (FIB). Sections of bulk Xe irradiated material were annealed in a quartz tube furnace under inert atmosphere at $800^{\circ} \mathrm{C}$ for 20 min prior to FIB sectioning. FIB lamellae of bulk Xe irradiated material were also cut for TEM in situ annealing using a DENS Solutions Wildfire heating stage in the temperature range $100-500{ }^{\circ} \mathrm{C}$.

These FIB lamellae were examined using a double Cs corrected ARM 200F transmission electron microscope operating at $200 \mathrm{kV}$ in both TEM and STEM mode.

\section{Results}

\subsection{TEM results at room temperature}

Figure 1 shows two bright field (BF) TEM micrographs of Xe latent tracks in the same material in cross-section (a) and plan view (b). Individual tracks have a rectangular cross-section and consists of sections misaligned at a slight angle to the incident radiation. The discontinuity is demonstrated in the BF TEM micrograph (Fig. 2) showing one such latent track along the ion direction. It is observed that the sections for the latent tracks are approximately $25 \mathrm{~nm}$ length. The angular displacement at the discontinuity between the two sections is about 9 degrees which is in agreement with the fact that the tetragonal columns are discontinuous because of $9^{\circ} a-c$ angle mismatch with monoclinic phase. The contrast due to the strain at the boundary between the tetragonal column and the monoclinic matrix, due to the lattice mismatch, is clearly visible. The crystallographic structure for the two phases are shown for a planar view in the HAADF STEM micrograph (Fig. 3). The insets show the simulated atomic columns which are overlaid for comparison and hence confirming both the tetragonal phase for the latent track and the monoclinic phase for the matrix. It is clear that the tetragonal phase was found to remain stable at room temperature and also after preparation of the FIB lamellae using Ga ions. The stability of the tetragonal phase was also not compromised under electron irradiation in the TEM.

\subsection{TEM analysis of annealed material}

In an attempt to verify the $(t \rightarrow m)$ transformation temperature, bulk irradiated zirconia was subjected to annealing at $800^{\circ} \mathrm{C}$ for $20 \mathrm{~min}$. The tetragonal phase in the tracks had transformed back to the monoclinic phase leaving a line of defect clusters as shown in the bright field TEM image (Fig. 4). The nature of these defects was not determined due to their small size.
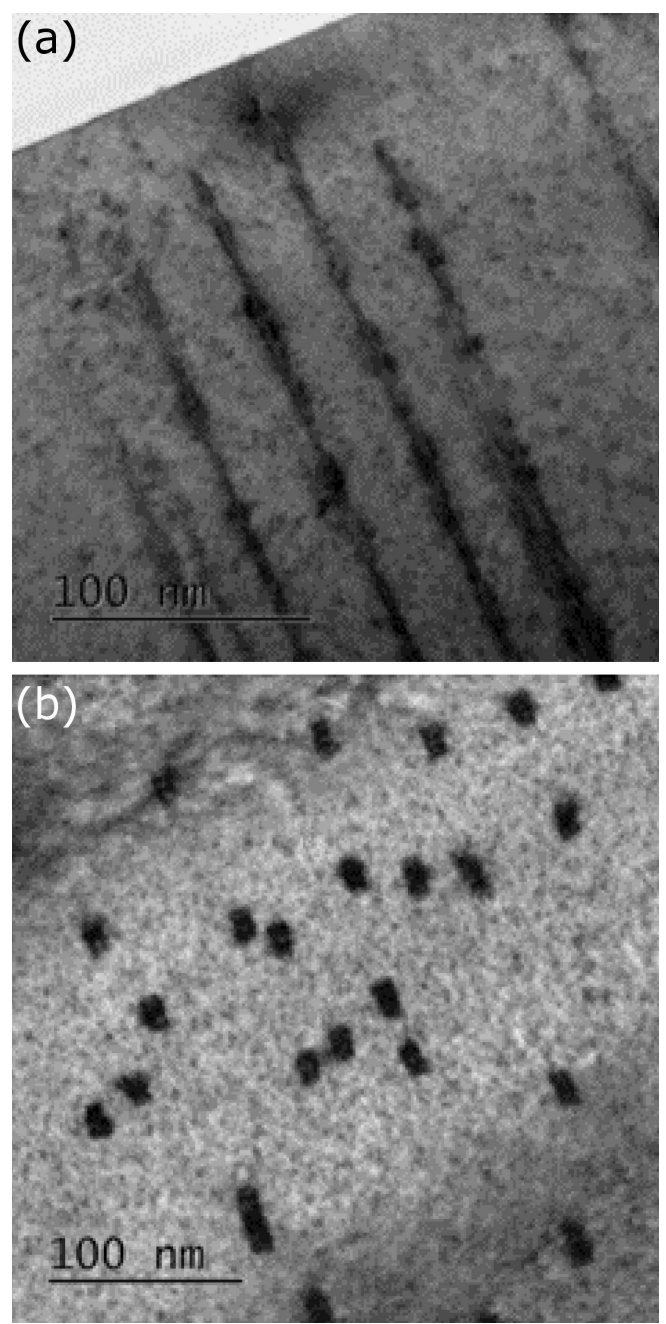

Fig. 1. (a) BF TEM micrographs in cross-section and (b) plan view of $167 \mathrm{MeV} \mathrm{Xe}$ latent tracks in natural $\mathrm{ZrO}_{2}$.

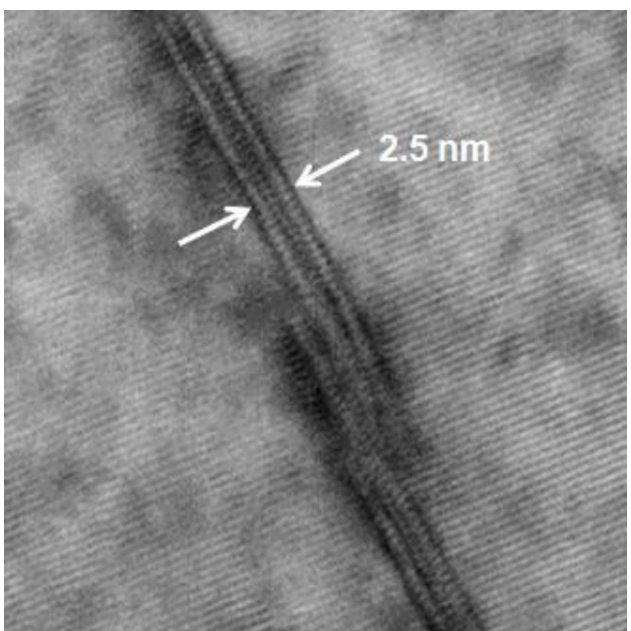

Fig. 2. BF TEM micrograph (in cross-section) of a single ion track showing the discontinuity. The image was taken close to the $[100]$ zone axis of the monoclinic phase and the visible fringes are the (001) planes. 


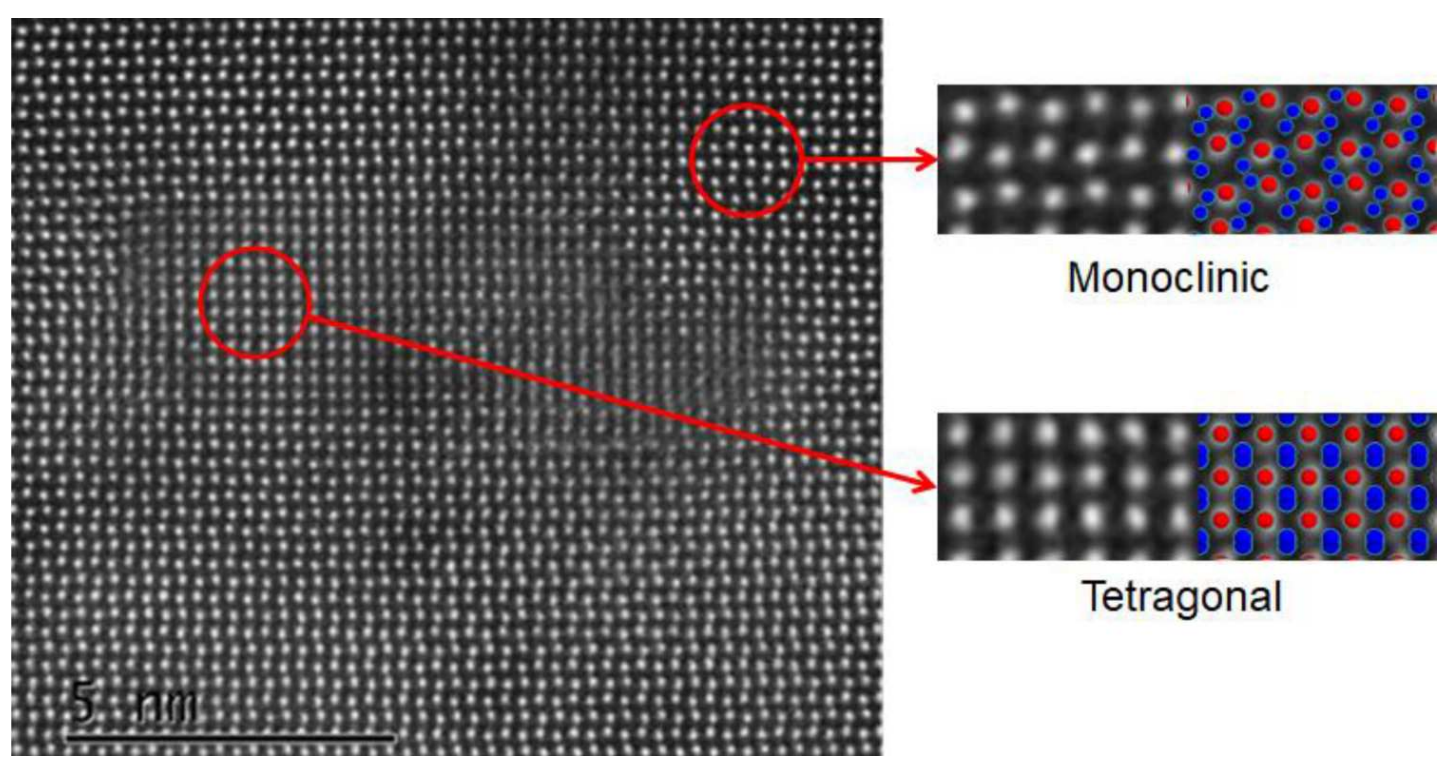

Fig. 3. (a) HAADF STEM micrographs for a single $167 \mathrm{MeV}$ Xe latent track in planar view, (b) atomic simulation for the two phases shown in (a) with $\mathrm{Zr}$ columns red and $\mathrm{O}$ columns blue. O columns are not visible in the HAADF image.

In the case of bulk material, the transformation stress and volume increase is reported to be relieved by microtwinning of the material [2]. However, due to the small size of the tetragonal inclusions, twinning is not expected for the studied system and indeed no evidence of twinning was observed. Further to the furnace annealing of the bulk material, controlled annealing studies of the FIB lamellae were performed in the TEM using an in situ heating holder. It was observed that the reverse phase transformation $(t \rightarrow m)$ of the tracks occurred in the range $350-500^{\circ} \mathrm{C}$ resulting in a line of defects as observed for the furnace annealing of the bulk material. The in situ transformation temperature range is well below the expected bulk material temperature value of $700^{\circ} \mathrm{C}$. The transformation temperature for the tetragonal tracks can be explained in terms of the sub-critical volume for the tetragonal phase and the subsequent release of strain energy upon the back transformation to the monoclinic phase. The monoclinic phase is still the thermodynamically preferred phase between 350 and $500^{\circ} \mathrm{C}$ where the back transformation was observed. Given this, and the fact that the surrounding monoclinic phase exerts significant stress on the tetragonal inclusion, it is likely that the activation energy for the backtransformation is sufficiently lowered that the transformation may be initiated at $350^{\circ} \mathrm{C}$.

\section{Conclusion}

In this work we have observed single ion tracks by transmission electron microscopy which were produced under low fluences and using relatively light ions $(\mathrm{Xe})$ in monoclinic zirconia $\left(\mathrm{ZrO}_{2}\right)$. The tracks were analyzed in the TEM and found to consist of the tetragonal phase. Back transformation to the monoclinic phase

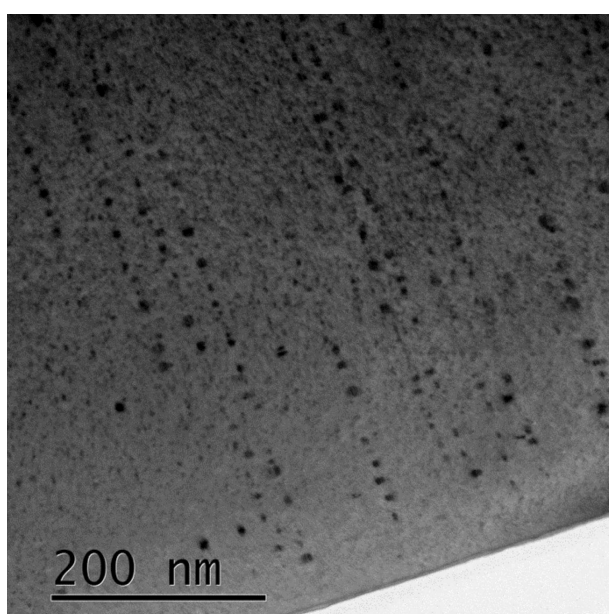

Fig. 4. BF TEM image showing lines of defects in zirconia after annealing of latent tracks.

started upon heating to $350{ }^{\circ} \mathrm{C}$ and complete transformation was observed at $500^{\circ} \mathrm{C}$. The low temperature at which the back transformation occurred compared to that in the literature is believed to be due to high levels of stress in the tetragonal phase due to the adjacent monoclinic bulk material. To our knowledge, this is the first report of stabilized tetragonal zones within individual latent tracks in the non-overlapping regime for zirconia.

\section{Acknowledgments}

The authors would like to thank Dr. Marina Zakhozheva and Diederik Morsink from DENS Solutions for the use of the Wildfire in situ heating holder. 


\section{References}

[1] J. Chevalier, L. Gremillard, A.V. Virkar, D.R. Clarke, J. Am. Ceram. Soc. 92, 1901 (2009).

[2] M. Mamivand, M.A. Zaeem, H.E. Kadiri, L.Q. Chen, Acta Mater. 61, 5223 (2013).

[3] J.E. Bailey, Proc. R. Soc. 279, 395 (1964).

[4] R.C. Garvie, J. Phys. Chem. 69, 1238 (1965).

[5] A. Suresh, M.J. Mayo, W.D. Porter, J. Mater. Res. 18, 2912 (2003).

[6] K.E. Sickafus, H. Matzke, T. Hartmann, K. Yasuda, J.A. Valdez, P. Chodak, M. Nastasi, R.A. Verrall, J. Nucl. Mater. 274, 66 (1999).
[7] B. Schuster, M. Lang, R. Klein, C. Trautmann, R. Neumann, A. Benyagoub, Nucl. Instrum. Meth. B 267, 964 (2009).

[8] W. Jozwik, J. Jagielski, G. Gawlik, P. Jozwik, R. Ratajczak, G. Panczer, N. Moncoffre A. Wajler, A. Sidorowicz, L. Thomé, Phys. Chem. Miner. 43, 439 (2016).

[9] J. Jagielski, L. Thomé, A. Chartier, O. Dorosh, C. Mieszczynski, I. Jozwik, Nucl. Instrum. Meth. Phys. Rev. B 435, 2 (2017). 